\title{
Addictive agents and intracranial stimulation: Self- stimulation under morphine, amphetamine, and chlorpromazine
}

\author{
DEBRA J. MAGNUSON, CAROL J. TADEUSIK, and LARRY D. REID \\ Bradley University, Peoria, Mlinois 61606
}

\begin{abstract}
Rats fixed with chronically indwelling electrodes for intracranial stimulation (ICS) of the hypothalamus pressed daily for ICS. Some rats were exposed to ICS of 50, 75, and 100 microA (60-Hz sine waves of .3 sec), while others responded for ICS of 40 microA or less. Exposure to the higher ICS reduced pressing previously sustained by lower ICS. Morphine, amphetamine, and chlorpromazine were tested for their effects on pressing for both groups. Similar drug effects were found for the lower and higher intensity groups.
\end{abstract}

In rats, morphine can accelerate pressing for lateral hypothalamic intracranial stimulation (ICS). Doses of $10 \mathrm{mg} / \mathrm{kg}$ of morphine sulfate, for example, lead to increased pressing for ICS from 3 to $5 \mathrm{~h}$ after a subcutaneous injection (Adams, Lorens, \& Mitchell, 1972; Bush, Bush, Miller \& Reid, 1976). Such increased pressing is not a product of potential increased acitivity that might have occurred with this regimen of dosing and testing (Lorens \& Mitchell, 1973), nor a product of morphine's analgesic properties (Farber \& Reid, 1976). Increased pressing seems to parallel a positive affective state as indexed by rats moving to the place where they had previously experienced morphine (Rossi \& Reid, 1976). A reasonable hypothesis is that morphine can potentiate activity in the medial forebrain bundle and that this potentiated activity is reflected in increased self-stimulation, in movement of rats to the place of morphine experience and in subjective reports of euphoria among human users of opiates (McAuliffe \& Gordon, 1974).

Terando, Mirza, Zipnick, Overmeir, Rossi and Reid (Note 1) demonstrated greater acceleration of pressing due to morphine among rats that had experienced high intensities of ICS compared to rats that had not experienced the higher intensities. The high-intensity ICS was about 100 microA of $60-\mathrm{Hz}$ sine waves and was sufficiently intense to lead to disruption of pressing for ICS at lower intensities (about 25 microA). It seemed from the data of Terando et al. that a lesion, probably induced by the higher intensities of ICS, somehow augmented morphine's capacity to facilitate pressing. Results of Terando et al. are based on a small number of rats and, therefore, may not be as substantial as they appear. Consequently, this study attempted to answer the question of whether or not morphine

This work was supported by Grant DA 01049 from the National Institute of Drug Abuse, DHEW. This work is included in a Senior Honor's Thesis of the first author. We thank Nello Rossi for his assistance. produces differential changes in self-stimulation in subjects having sustained or not having sustained highintensity ICS. Amphetamine and chlorpromazine were also tested.

\section{METHOD}

\section{Subjects}

Subjects were 10 experimentally naive male adult SpragueDawley rats. Each was fixed, in standard ways, with a chronically indwelling bipolar platinum electrode (Plastic Products) insulated with Teflon except at the cross-section of the electrode tips. They were housed individually with food and water always available.

\section{Apparatus}

Testing was done in a Plexiglas box $(24 \times 30 \times 35 \mathrm{~cm})$ fitted with a standard lever, the depression of which yielded a single train of ICS. The ICS was $60-\mathrm{Hz}$ sine waves of $.3 \mathrm{sec}$ of varied intensities, with 100 microA (peak to peak) being the highest intensity delivered. ICS was delivered by way of light, flexible leads attached to a slip ring allowing subjects unhampered movement. Barpresses during any given 2-min interval were recorded automatically.

\section{Procedure}

Rats were tested daily after surgery of fixing electrodes (recovery from surgery spanned 5 days) and after a few days of initial testing for self-stimulation. During the first sessions, three intensities were selected for each subject. The first three intensities, as well as all initial ICS, were less than 50 microA. The selected low ICS was just above the lower threshold for intracranial reinforcement and ranged across subjects from 8 to 16 microA. The high ICS sustained rapid pressing and ranged from 18 to 40 microA. The medium ICS was about midway between low and high ICS and ranged from 12 to 35 microA.

After selecting intensities, a subject was allowed to press daily at each intensity for $2 \mathrm{~min}$, starting with low ICS and ending with high ICS. Each 2-min measurement was preceded by a period during which a subject pressed for at least 10 ICSs to acquaint it with the intensity to be used during a measurement.

After 10 daily sessions to stabilize pressing, subjects were assigned to one of two groups, five subjects per group. For one group, daily sessions with ICS continued at the previously selected intensities. For the other group, three higher intensities, 50,75 , and 100 microA, were introduced. During three daily 
sessions, the group of only lower intensities was tested at their originally selected intensities and subsequently tested for three additional 2-min periods at their originally selected high ICS; for example, Subject 682 was run at 12,18 , and 22 microA, respectively, during three 2 -min periods and then at 22 microA during three additional 2-min periods. Correspondingly, the high-intensity group was tested at their originally selected intensities and subsequently at 50,75 , and 100 micro $A$.

After the days of higher intensities, subjects were again tested with only three intensities of ICS. The group of lower intensities was tested with each of the originally selected intensities. The group of higher intensities continued with 50, 75, and 100 microA, the new low, medium, and high ICSs. Following 3 days with the three intensities, tests were begun to assess the effects of drugs on pressing. Subjects continued daily opportunities to press for $2 \mathrm{~min}$ at each of the three intensities; for one group, lower intensities; for the other group, higher intensities. During some days, subjects given opportunities to press were under the influence of drugs.

The following doses and times after dosing were assessed: $2 \mathrm{mg} / \mathrm{kg}$ chlorpromazine injected intraperitoneally $45 \mathrm{~min}$ before testing, $.75 \mathrm{mg} / \mathrm{kg}$ amphetamine injected intraperitoneally $30 \mathrm{~min}$ before testing, and $10 \mathrm{mg} / \mathrm{kg}$ morphine sulfate given subcutaneously $4.5 \mathrm{~h}$ before testing. All drugs were carried in physiological saline and saline was the placebo. Each drug was tested once in each rat across a 12-day series of daily sessions broken into three 4-day sequences. Each sequence was a day's session of base (no injection), a session of placebo, a session under the drug, and another session of base. To counterbalance sequential effects, each drug was tested at least once in each position in the order of testing, and the same sequences were used for both groups.

Subsequent to tests, rats' brains were taken following standard procedures. Frozen 75-micron sections of brain were treated as photographic negatives and enlarged photographs made to aid in determination of the ICS site.

\section{RESULTS}

The effects of introducing the higher intensities of ICS on pressing for the originally selected intensities are summarized in Figure 1. The means of Figure 1 are from the tests just before introduction of higher ICS and from those 3 days later. The two groups pressed similarly before introduction of higher intensities (two left panels of Figure 1). The group not exposed to the higher intensities continued to press about the same on the first and last test (bottom panels of Figure 1). Pressing for the originally selected low and medium ICS was reduced considerably by introduction of higher intensities; this is depicted in Figure 1, top panels.

Using data germane to Figure 1 , a 2 by 2 by 3 analysis of variance (ANOVA) having repeated measures was done, with factors of the two groups, before-after introduction of procedures, intensities of ICS, and the interactions. Of course, the factor associated with intensity of ICS was reliable $[F(2,16)=18.0, p<.01]$. Groups was not a reliable source of variance but the factor associated with before-after the procedures was reliable $[F(1,8)=13.6, p<.01]$. The interesting interaction of Groups by Before-After was reliable $[F(1,8)=24.1, p<.01]$. The Before-After by Intensity interaction was also reliable $[\mathrm{F}(2,16)=10.3, \mathrm{p}<.01]$,

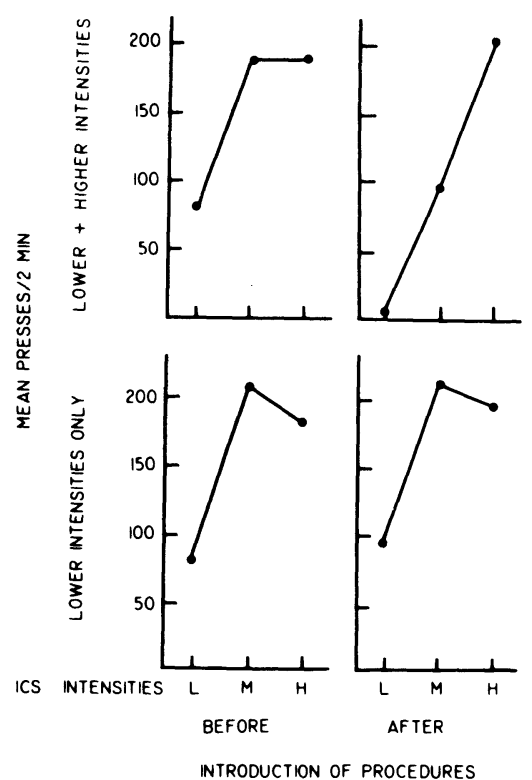

Figure 1. Pressing for three standard intensities of ICS (a low, medium, and high intensity) before and after introduction of higher intensities of ICS.

as well as the triple interaction of Groups by BeforeAfter by Intensity $[F(2,16)=7.6, p<.01]$. Other sources of variance did not meet standards of statistical significance.

Student $t$ tests for correlated measures across comparable scores of the group of only low intensity (Figure 1, bottom panels) yielded no value indicating reliable shifts in pressing across days of testing. Student $t$ tests between scores of subjects exposed to the higher intensities confirmed that pressing was subsequently reduced at low and medium ICS [t(4) for low ICS = 7.57 ; $t(4)$ for medium ICS $=3.96$, ps $<.01]$. In summary, data support the conviction that introducing intensities of 50,75 , and 100 microA nearly abolishes previously stable responding for ICS of about 15 microA and reduces pressing for higher intensities.

A 2 by 2 by 3 by 3 ANOVA having repeated measures was performed to compare baseline performances just before placebo injections to performances under placebo injections, with factors associated with the two groups, base-placebo, the three instances of base-placebo testing, and the three intensities. No factor of this ANOVA was a statistically significant effect, except those associated with the variable of intensity. The main effect comparing scores of base and placebo, for example, yielded an $F(1,8)=1.4, p>.25$. Consequently, effects seen with drug injections are likely due to the drugs, not to the injections themselves. As would be expected, the factor associated with intensity was a reliable source of variance $[F(2,16)=11.0, p<.01]$. Also, the interaction of Group (subjects of lower and higher intensities) by Intensities (low, medium, and high intensities of testing) 
was a reliable effect $[F(2,16)=12.4, p<.01]$. Since mean pressing scores for the respective medium and high intensities of the two groups are nearly the same, this last effect is due almost entirely to the difference in the groups' pressing at their low intensities. Rats of original intensities pressed slowly at their low intensity, but rats of the new higher intensities pressed rapidly at their new "low intensity" of 50 microA. The gist of this ANOVA is that groups differed with respect to pressing at their respective low intensities, but otherwise they were comparable prior to introduction of drugs. Therefore, any differential drug effects seen across their respective low intensities might be due to differences in baseline-placebo performances. Ratio scores of drug to placebo have the effect of reducing variance attributable to intensity; therefore, they were used to assess drug effects.

Figure 2 depicts the results of testing with drugs by showing ratio scores of drug to the immediately preceding placebo. Any score greater than one indicates that the drug accelerated pressing for ICS. Using scores collapsed across intensities, it can be concluded that amphetamine and morphine produced average increments in pressing, but not reliably $[\mathrm{t}(9)=1.27$, $t(9)=1.58$, respectively, ps $>.10]$. Chlorpromazine produced reliable decreases in pressing $[\mathrm{t}(9)=-3.98$, $\mathrm{p}<.011$. Separate 2 by 3 ANOVAs for each panel in Figure 2 did not support the hypothesis of differential drug effects across the two groups for morphine and chlorpromazine (all ps $>.20$ ).

The ANOVA for amphetamine yielded the following values: $F(1,8)$ for group $=2.3, p<.20 ; F(2,16)$ for intensity $=2.8, \mathrm{p}<.10$. The correlation between numbers of presses under placebo and the measures of amphetamine's effect (the ratios of placebo to drug) at the low intensity is $-.79(\mathrm{p}<.01)$, indicating that the smaller the placebo score, the more the increment in pressing under amphetamine. Since the subjects of only lower intensities pressed at their low intensity less than subjects of higher intensities, any correction of the scores based on level of placebo responding would minimize differences. Since the ANOVA already indicates no reliable differences and since "correcting" the scores by taking into account placebo responding would lessen differences, the appropriate conclusion is that amphetamine did not produce reliable differential

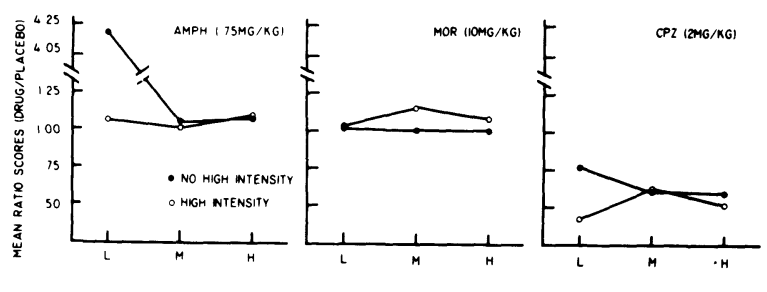

INTENSITIES OF ICS

Figure 2. Mean ratio scores of press rates reflecting effects of amphetamine, morphine, and chlorpromazine. effects in the groups tested at lower and higher intensities.

Even though the average facilitation of pressing under amphetamine and morphine was not marked, individual rats did show strongly facilitated pressing under one or the other of the two drugs. Rat 699 , for example, had a ratio score of 1.56 at medium ICS under morphine and Rat 687 had a ratio score of 1.30 at medium ICS under amphetamine. A single score was calculated for each subject with each drug to reflect that subject's response to the drug. That calculated score was the mean ratio of drug to placebo across intensities of ICS. Then a correlation coefficient was calculated between rats' responsiveness to each pair of drugs, yielding, of course, three correlations: amphetamine to morphine, -.61 (p about .5); amphetamine to chlorpromazine,.36; and, morphine to chlorpromazine, -.18 .

It was not possible to tell by inspection of enlarged (x10) photographs of brain sections which electrode sites sustained the higher intensities and which did not. Also, there was no apparent relationship between site of ICS and whether or not morphine or amphetamine led to increased pressing.

\section{DISCUSSION}

This data as well as that of Terando et al. (Note 1) lead to the strong conclusion that even a relatively brief introduction of ICS between 50 and 100 microA is sufficient to reduce pressing previously sustained by low intensities. Becker and Reid (Note 2) demonstrated that $20 \mathrm{sec}$ of $20-$ microA $60-\mathrm{Hz}$ sine waves, programmed periodically, reduced pressing for lowintensity ICS. Consequently, the parameters of ICS used in many studies (e.g., Ellman, A ckerman, Bodnor, Jackler, \& Steiner, 1975) can modify the tissue of ICS. This effect could confound the conclusions that might be drawn, and it was thought that modification of tissue by the higher intensities of ICS might, in turn, modify drug action. This study, however, failed to find such differential drug action. Although these data do not allow the conclusion of different drug effects between high- and lowintensity groups, it is not unreasonable to suppose that differential effects might be obtained in other tests.

There are considerable individual differences in magnitude of responsiveness to morphine with respect to pressing for hypothalamic ICS both across and within studies (Adams et al., 1972; Terando et al., Note 1). Even when parameters of ICS are similar, some subjects show marked acceleration of pressing under morphine, while others do not. Furthermore, the acceleration of pressing due to morphine and due to amphetamine seems to involve different processes, since across subjects they are negatively correlated. The stereotypic behaviors produced by morphine and by amphetamine, although grossly similar, also seem to be produced by different processes (Ayhan \& Randrup, 1972; Villarreal, Guzman, \& Smith, 1973). Understanding individual differences with respect to reactivity to morphine is, of course, an interesting problem.

Between the last self-stimulation test and the procedures for determining electrode site, the rats had 15 days of opportunity to choose between a sweetened morphine solution and water in their home cages. Rats showing the greatest acceleration of pressing for ICS under morphine were those that initially drank the most morphine solution (rho between ratio of morphine/placebo pressing for medium ICS and milliliters consumed across initial 3 days $=.86, p<.01$ ) (Magnuson, Note 3). Although this observation suggests an important 
relationship between degree of facilitation of pressing for ICS and potential avidity with respect to morphine, there are a number of problems in determining degree of facilitation. The level of pressing during baseline or placebo is a factor in determining the degree of acceleration under a drug; consequently, numbers indexing degree of acceleration can vary without telling much about morphine's actions. Therefore, conclusions based on degree of acceleration of pressing for ICS, including those drawn here, are subject to misinterpretation. For strong conclusions to be drawn from studies assessing magnitude of acceleration in pressing for ICS, site of stimulation and intensity of ICS must be carefully controlled.

\section{REFERENCE NOTES}

1. Terando, L., Mirza, N., Zipnick, J., Overmier, M., Rossi, N., \& Reid, L. Addictive agents and intracranial stimulation (ICS): Daily morphine, self-stimulation, and parameters of ICS. In preparation.

2. Becker, B. M., \& Reid, L. D. Changes in pressing for intracranial stimulation (ICS) after prolonged ICS. In preparation.

3. Magnuson, D. J. Intracranial self-stimulation under the influence of morphine, amphetamine, and chlorpromazine. Unpublished Senior Honor's Thesis, Bradley University, 1976.

\section{REFERENCES}

Adams, W. J., Lorens, S. A., \& Mitchell, C. L. Morphine enhances lateral hypothalamic self-stimulation in the rat. Proceedings of the Society of Experimental Biology and Medicine, 1972, 140, 770-771.
AYhan, I. H., \& RANDRUP, A. Role of brain noradrenaline in morphine-induced stereo-typed behavior. Psychopharmacologia, 1972, 27, 203-212.

Bush, H. D., Bush, M. F., Miller, M. A., \& Reid, L. D. Addictive agents and intracranial stimulation: Daily morphine and lateral hypothalamic self-stimulation. Physiological Psychology, 1976, 4, 79-85.

Ellman, S. J., Ackerman, R. F., Bodnor, R. J., Jackier, F., \& STEIner, S. S. Comparison of behaviors elicited by electrical brain stimulation in dorsal brain stem and hypothalamus of rats. Journal of Comparative and Physiological Psychology, 1975, 88, 816-828.

FARBER, P. D., \& REID, L. D. Addictive agents and intracranial stimulation (ICS): Daily morphine and pressing for combinations of positive and negative ICS. Physiological Psychology, 1976, 4, 262-268.

LoRENS, S. A., \& Mitchell, C. S. Influence of morphine on lateral hypothalamic self-stimulation in the rat. Psychopharmacologia, 1973, 32, 271-277.

McAuliffe, G. E., \& Gordon, R. A. A test of Lindesmith's theory of addiction: The frequency of euphoria among longterm addicts. American Journal of Sociology, 1974, 79, 795-840.

Rossi, N. A., \& REID, L. D. Affective states associated with morphine injections. Physiological Psychology, 1976, 4, 269-274.

Villarreal, V. E., Guzman, M., \& Smith, C. B. A comparison of the effects of d-amphetamine and morphine upon the locomotor activity of mice treated with drugs which alter brain catecholamine content. Journal of Pharmacology and Experimental Therapeutics, 1973, 187, 1-7.

(Received for publication August 10, 1976.) 